BASIC SCIENCE ARTICLE

\title{
Heme oxygenase-1 deficiency promotes severity of sepsis in a non-surgical preterm mouse model
}

\author{
Kazumichi Fujioka ${ }^{1}$, Flora Kalish ${ }^{1}$, Hui Zhao ${ }^{1}$, Ronald J. Wong ${ }^{1}$ and David K. Stevenson ${ }^{1}$
}

\begin{abstract}
BACKGROUND: Sepsis in preterm infants is associated with systemic inflammatory responses. The stress-response protein heme oxygenase-1 (HO-1) has protective anti-inflammatory properties. Recently, we reported a protective role of HO-1 using our nonsurgical cecal slurry (CS) model in wild-type (WT) mouse pups. Here, we extend these findings to investigate the association of HO-1 deficiency with sepsis severity.

METHODS: Adapting the Wynn model, we induced sepsis in 4-day-old HO-1-deficient (HO- $1^{+/-}$, Het) pups to determine if $\mathrm{HO}-1$ deficiency affected survival rates at the LD40 $(2.0 \mathrm{mg} / \mathrm{g})$ of WT pups. To see if HO-1 induction affected sepsis severity, we gave 30 $\mu \mathrm{mol}$ heme/kg subcutaneously to 3 -day-old mice $24 \mathrm{~h}$ prior to sepsis induction.

RESULTS: Post-sepsis induction, Het pups had a mortality of $85.0 \%(n=20)$ and increased expression of the pro-inflammatory gene in the livers and affected hematologic profiles. Heme treatment $24 \mathrm{~h}$ prior to sepsis induction significantly increased liver HO activity, reduced mortality to $24.5 \%(n=17)$, attenuated inflammatory responses, reduced spleen bacterial counts, and significantly increased peripheral neutrophils.

CONCLUSIONS: A partial deficiency in HO-1 increased the progression and mortality in sepsis. Furthermore, induction of HO-1 significantly reduced the mortality even in Het pups. Thus, we conclude that HO-1 plays an important role in the protection against preterm sepsis.
\end{abstract}

Pediatric Research (2018) 84:139-145; https://doi.org/10.1038/s41390-018-0028-6

\section{INTRODUCTION}

Despite recent advances in neonatal intensive care, sepsis is still a leading cause of mortality in preterm infants. Deaths due to sepsis are much higher in preterm infants, especially in those born with extremely low birthweights (ELBW), than in term infants, and may be related to immature immune systems. ${ }^{1-3}$ Although the pathophysiology of preterm sepsis has not been well elucidated, multiple precipitating factors such as inflammation, oxidative stress, and apoptosis have been hypothesized to play key roles. Heme oxygenase $(\mathrm{HO})$ is the rate-limiting enzyme in the pathway of heme degradation, which results in the equimolar generation of free iron, biliverdin, and carbon monoxide (CO). ${ }^{4,5}$ Its inducible isoform, HO-1, also known as heatshock protein-32, possesses multiple cytoprotective properties through its regulation of antiinflammatory, antioxidative, and anti-apoptotic processes. ${ }^{6}$ Several studies have investigated its role in the pathophysiology of sepsis in mice ${ }^{7-10}$ and humans. ${ }^{11,12}$ However, most research has been performed using adult rodents; and therefore, these findings may not be extrapolated to newborn pups.

To this end, we have applied our established non-surgical mouse model ${ }^{13}$ of preterm sepsis, which is an adaptation of the cecal slurry (CS) method first proposed by Wynn et al. ${ }^{2}$ and then modified by Starr et al. ${ }^{14}$ and Steele et al., ${ }^{15}$ to 4-day-old wild-type (WT) mouse pups, which at this age are immunologically equivalent to preterm human infants. ${ }^{16}$ Using this model, we have demonstrated a clear dose-dependent mortality, bacterial colonization in all examined organs, significant weight loss in pups, hematological changes, and increases in the expression of pro-inflammatory cytokine, pattern recognition receptor, and other immune-related genes. ${ }^{13}$ Moreover, we also observed that the induction of HO-1 reduced the progression and severity of sepsis, and hypothesized a protective role of HO- $1 .{ }^{13}$ Thus, the objective of this study was to investigate whether a partial deficiency in HO-1 can promote the severity of preterm sepsis by altering the immune response.

\section{MATERIALS AND METHODS}

Animals

Adult FVB/n WT $\left(\mathrm{Hmox}^{+/+}\right)$breeders were obtained from Charles River Laboratories (Wilmington, MA) and used as the source of WT pups. Adult $\mathrm{HO}-1$ heterozygote $\left(\mathrm{Hmox}^{+/-}\right.$, Het) breeder pairs from our mouse colony were used as the source of Het pups. The original HO-1 knockout $\left(\mathrm{Hmox} 1^{-1-}\right)$ mouse strain has a targeted deletion of a large portion of Hmox 1 gene and was created on a C57BL/ 6 background. To establish our FVB/n Het colony, C57BL/6 $\mathrm{Hmox} 1^{-1-}$ mice were backcrossed with $\mathrm{FVB} / \mathrm{n}$ WT mice for $>6$ generations. ${ }^{17}$ All pups were housed with their mothers throughout the course of the study. For each experiment, the pups were randomized on an individual basis within each litter. In addition, to eliminate any litter bias effects, we used at least three different litters for each experimental group. Stanford University's Institutional Animal Care and Use Committee approved the study (Protocol \# 30910). 


\section{Genotyping}

Genomic DNA was isolated from tail clippings using the Tissue DNeasy kit (Qiagen, Valencia, CA) and typed by PCR. For $\mathrm{Hmox} 1^{+/+}$and $\mathrm{Hmox}^{+/-}$screening, two sets of primers specifically designed for WT and Het genotyping were used as previously described. ${ }^{17}$

\section{Preterm Sepsis Model}

As in our previous report, ${ }^{13}$ a single stock CS solution was prepared from the cecums harvested from adult (8 to 10-wk-old) female FVB/n mice (fed Teklad Rodent Diet \#2018, Envigo, Hayward, CA) according to the methods of Starr et al., ${ }^{14,15}$ and then stored at $-80^{\circ} \mathrm{C}$ in $1-\mathrm{mL}$ aliquots until use for all studies. To induce sepsis, we applied the CS model established by Wynn et al., ${ }^{2}$ but we used our stored stock (versus fresh) CS solution to induce sepsis. In brief, 4-day-old pups of both genotypes (WT and Het) were given $2.0 \mathrm{mg}$ of CS/g bodyweight (BW) intraperitoneally (IP), the LD40 established for WT pups in our previous report, ${ }^{13}$ and then closely monitored daily for health and survival for up to 7 days. The CS used in these studies was from the same stock used in our previous study, which was used to establish the LD40. ${ }^{13}$

Heme induction

A stock solution of 4.0-mM methemalbumin (MHA) was prepared as previously described ${ }^{18}$ using hemin and bovine serum albumin (BSA) (Sigma-Aldrich, St Louis, MO). At day 3 of age, the pups were administered vehicle or MHA $(30-\mu \mathrm{mol} / \mathrm{kg} \mathrm{BW})^{18,19}$ in a volume of $\sim 25 \mu \mathrm{L}$ subcutaneously (SC) at the scruff of the pup's neck using a $0.5-\mathrm{mL}$ syringe fitted with a 30 -ga needle.

Bacteriologic examination

Because the liver and spleen were the two main organs affected after sepsis induction in our previous study, ${ }^{13}$ and that the spleen is the primary site of bacterial localization and endotoxin during infection, ${ }^{20}$ and the source of circulating pro-inflammatory cytokines, ${ }^{21}$ we focused on these tissues for detailed analyses.

Twenty-four hours after CS administration (or at 5 days of life), the livers and spleens were removed, homogenized, and diluted serially 1:10 in PBS. The diluted homogenates were streaked onto agar plates containing brain heart infusion (BHI) broth, the plates were incubated at $37^{\circ} \mathrm{C}$ for $24 \mathrm{~h}$, and colony forming units (CFU) of bacteria per organ were quantified as described previously. ${ }^{14}$

HO activity $^{19}$

After sacrifice, the livers and spleens were harvested and rinsed with ice-cold $0.1 \mathrm{M} \mathrm{KPO}_{4}$. One-hundred milligrams of tissue were diluted $10 \times$ with $0.1 \mathrm{M} \mathrm{KPO}_{4}$. The tissues were then sonicated at $50 \%$ power with a Microson Ultrasonic Cell Disruptor (Misonix, Farmingdale, NY). Liver sonicates $(20 \mu \mathrm{L}$ : representing $2 \mathrm{mg}$ of tissue) were incubated with equal $(20 \mu \mathrm{L})$ volumes of NADPH (4.5 $\mathrm{mM})$ and MHA (150- $\mu \mathrm{M}$ heme/15- $\mu \mathrm{M} \mathrm{BSA})$ for $15 \mathrm{~min}$ at $37^{\circ} \mathrm{C}$ in 2$\mathrm{mL}$ CO-purged septum-sealed vials. Reactions were terminated with the addition of $5 \mu \mathrm{L}$ of $15 \%$ sulfosalicylic acid. The amount of $\mathrm{CO}$ in the vial headspace was determined by gas chromatography with a Reduction Gas Analyzer (RGA-2, Peak Laboratories LLC, Mountain View, CA). HO activity was calculated as $\mathrm{pmol} \mathrm{CO} / \mathrm{h} / \mathrm{mg}$ fresh weight (FW) and expressed as \% $\mathrm{HO}$ activity of age-matched controls.

\section{Hematology}

Twenty-four hours (i.e., 4 days of life) post-heme administration and at $24 \mathrm{~h}$ (i.e., 5 days of life) post-sepsis induction, the pups were sacrificed by decapitation and approximately $30 \mu \mathrm{L}$ of blood was immediately collected in Microtainer ${ }^{\circledR}$ Tubes containing EDTA (BD Sciences, San Jose, CA) for measurements of complete blood counts (CBCs) with automated differential method. All measurements were performed by the Diagnostic Laboratory in the Department of Comparative Medicine (Stanford, CA).
PCR-array (84 genes, mouse cytokines and chemokines)

Six hours after CS, approximately $5 \times 5 \times 1 \mathrm{~mm}$ pieces from freshly harvested livers and spleens were placed in liquid nitrogen and then stored at $-80^{\circ} \mathrm{C}$ until use. Total RNA was extracted according to standard laboratory procedures using the RNAeasy Mini Kit (Qiagen, Valencia, CA). cDNA was synthesized by use of a RT2 First Strand Kit (Qiagen). PCR array kits (Qiagen, catalog no. PAMM052Z) were used in this study. Real-time PCR was performed with RT2 Real-Time SYBR Green/ROX PCR Master Mix (Qiagen) on a Mx3005 Quantitative PCR System (Stratagene, Cedar Creek, TX). Optimal selections of internal housekeeping genes for analyses were automatically chosen by use of the web-based PCR Array analyses program (Qiagen). Fold changes in gene expression levels were then calculated using the $\Delta \Delta \mathrm{Ct}$ method (www. sabiosciences.com/pcrarraydataanalysis.php). Gene expression profiles of Het pups were then compared to those of WT pups as previously reported. ${ }^{13}$

\section{Statistical analyses}

Statistical analyses were performed using log-rank tests for Kaplan-Meier survival curves, unpaired Student's two-tailed $t$ tests for PCR array analyses, and Mann-Whitney tests or $\chi^{2}$ tests for comparisons between two groups. Differences were deemed statistically significant when $p<0.05$. Untreated WT control data from our previous paper ${ }^{13}$ were used for comparisons since those studies were performed concurrently with our present study and are not "historical".

\section{RESULTS}

Effect of a partial HO-1 deficiency on the severity of sepsis Mortality. As we have previously reported in our CS model, ${ }^{13}$ a CS dose of $2.0-\mathrm{mg} / \mathrm{g}$ was the LD40 for 4-day-old WT pups (Fig. 1, dotted line) and was used in this study to induce sepsis in Het pups. We found a mortality rate of $85.0 \%(n=20)$, which was significantly higher than that for WT pups $(p<0.01)$. In addition, at $24 \mathrm{~h}$ post-sepsis induction, BW gain in Het pups was less $(-5.7 \pm$ $2.6 \%, n=20)$ compared with WT pups $(-2.1 \pm 6.2 \%, n=21),{ }^{13}$ but was not statistically significant $(p=0.06)$.

Bacterial colonization. Twenty-four hours after CS administration, CFU counts in Het liver and spleen homogenates were $1.19 \pm$ $1.70 \times 10^{6}(n=8)$ and $47.6 \pm 78.8 \times 10^{6} \mathrm{CFU} / \mathrm{mL} \quad(n=8$, Fig. 2$)$, respectively, and equivalent to those we reported previously for WT pups. ${ }^{13}$

HO activity. Liver $\mathrm{HO}$ activity in 4-day-old Het pups $(212 \pm 28$ $\mathrm{pmol} \mathrm{CO} / \mathrm{h} / \mathrm{mg} \mathrm{FW}, n=11$, Fig. 3) was significantly lower than that of WT pups (302 $\pm 41 \mathrm{pmol} \mathrm{CO} / \mathrm{h} / \mathrm{mg} \mathrm{FW}, n=23, p<0.01)$, as

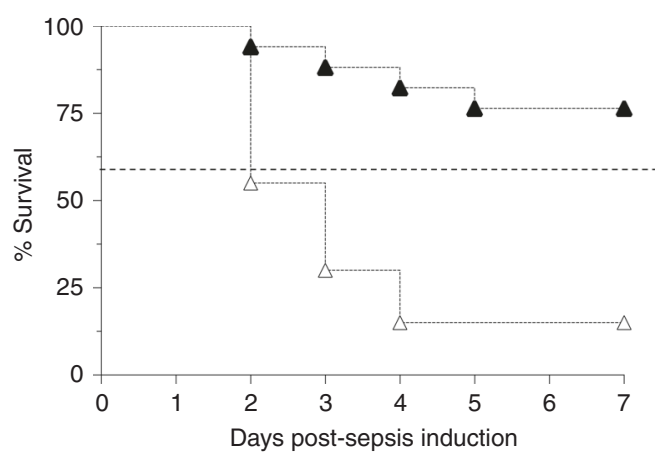

Fig. 1 Kaplan-Meier survival plots of 4-day-old Het pups given 2.0 $\mathrm{mg} / \mathrm{g}$ of CS IP $(\triangle, n=20)$ or pretreated with $30 \mu \mathrm{mol}$ of heme $/ \mathrm{kg} \mathrm{SC}$ $(\Lambda, n=17) 24 \mathrm{~h}$ prior to sepsis induction. Dotted line shows the LD40 (or 60\% survival) for WT pups as previously reported. ${ }^{13}$ Pups from at least three different litters were used in each group 
expected. Twenty-four hours post-sepsis induction, liver $\mathrm{HO}$ activity of Het pups increased 1.47-fold $(312 \pm 29 \mathrm{pmol} \mathrm{CO} / \mathrm{h} / \mathrm{mg}$ FW, $n=8$ ), but was significantly lower than that of the sepsisinduced (CS-only-treated) WT pups (485 $\pm 56 \mathrm{pmol} \mathrm{CO} / \mathrm{h} / \mathrm{mg} \mathrm{FW}$, $n=21, p<0.01)$.

Hematology. At baseline, white blood cell (WBC) and neutrophil counts (Table 1) in Het pups were similar from those we previously

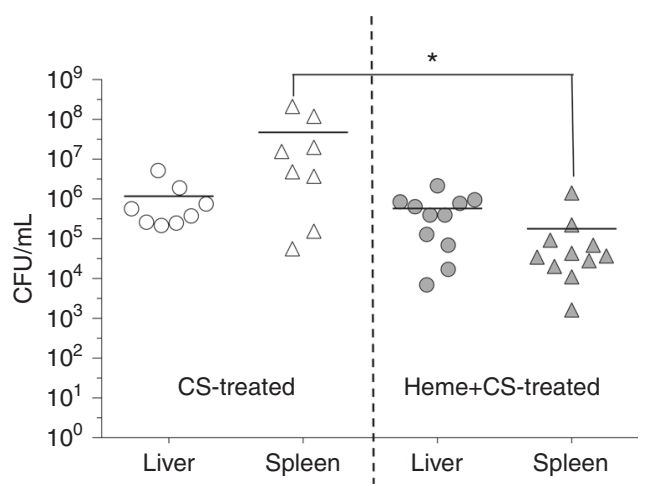

Fig. 2 CFU counts in the liver and spleen homogenates $24 \mathrm{~h}$ postsepsis induction in CS- $(n=8)$ and Heme+CS-treated $(n=11)$ Het pups; ${ }^{*} p<0.05$

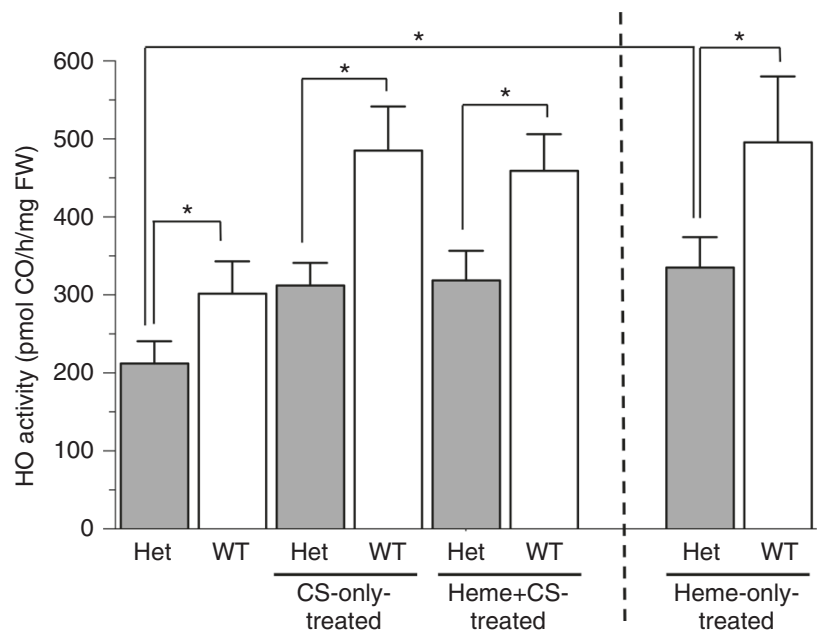

Fig. $3 \mathrm{HO}$ activity (pmol CO/h/mg FW) in Het (gray bars) and WT (white bars) livers in control pups ( $n=11$ and 25 , respectively), $24 \mathrm{~h}$ post-sepsis induction (CS-only-treated, $n=8$ and 21, respectively), pretreated with heme $24 \mathrm{~h}$ prior to sepsis induction (Heme+CStreated, $n=15$ and 10 , respectively), and $24 \mathrm{~h}$ post-heme induction (Heme-only-treated, $n=15$ and 10 , respectively); ${ }^{*} p<0.05$ reported for WT pups. ${ }^{13}$ However, lymphocytes and platelets were significantly lower $(p<0.05) .^{13}$

Twenty-four hours post-sepsis induction, WBCs, neutrophils, lymphocytes, and platelets in Het pups were comparable to those we previously found for similarly treated WT pups. ${ }^{13}$

All hematological parameters decreased after sepsis induction in both Het and WT pups compared to genotype-matched control pups.

Gene expression profiles. Using PCR arrays, we found that no genes in livers and spleen changed significantly in control Het compared with those previously reported for control WT pups. However, at $6 \mathrm{~h}$ post-sepsis induction, the expression of CD8a (-4.1-fold), CD14 (-4.8-fold), CCl5 (-9.6-fold), Ifng (-52.7-fold) in livers, and CD8a (-4.3-fold) in spleens in Het pups significantly decreased (Table 2). In contrast, TIr5 (6.9-fold) in livers and II6 (4.6fold) and Mpo (4.5-fold) in spleens significantly increased in control Het pups compared with WT pups.

Six hours post-sepsis induction, nine gene transcripts significantly increased and one significantly decreased in the livers of Het pups compared with control Het pups ( $p<0.05$, Table 3$)$. The increased genes were cytokines (Cxcl10, II23a, Tnf), patternrecognition receptors (Cd14, TIr2, NIrp3), and several other genes related to the immune response (Icam1, Myd88, Nfkbia). ${ }^{22,23}$ Interestingly, all upregulated genes (except /123a) were the same genes that were upregulated in CS-only-treated WT pups as previously reported. ${ }^{13}$ The only gene with decreased expression was chemokine receptor $\mathrm{Cx} C r 3$.

In contrast, 11 gene transcripts significantly increased and one significant decreased in spleens $(p<0.05$, Table 3$)$. Interestingly, these genes (except cytokines, Ccl12, I/1a, I/1b, I/6) were the same as those increased in livers, including cytokines (Cxcl10, Tnf), pattern-recognition receptors (Cd14, TIr2, NIrp3), and other genes related to the immune response (Icam1, Nfkbia). The only gene with decreased expression was the pattern recognition receptor TIr5.

Effect of HO-1 induction on the severity of sepsis Mortality. The administration of heme $(30 \mu \mathrm{mol} / \mathrm{kg} \mathrm{BW})$ to Het pups $24 \mathrm{~h}$ prior to sepsis induction significantly reduced mortality to $23.5 \%(n=17, p<0.01$, Fig. 1). Heme pretreatment also improved BW gain $(-0.6 \pm 8.4 \%, n=17)$, but was not statistically different from that of similarly treated Het pups $(p=0.07)$.

Bacterial colonization. CFU counts in the livers of Heme+CStreated Het pups $\left(5.82 \pm 6.27 \times 10^{5}, n=11\right)$ were comparable to Het pups treated with CS only. However, CFU counts in the spleens of Heme+CS-treated Het pups $\left(1.81 \pm 4.16 \times 10^{5}, n=11\right)$ were significantly decreased compared with that of the CS-onlytreated Het pups ( $p=0.001$, Fig. 2).

$\mathrm{HO}$ activity. To first determine if $\mathrm{HO}-1$ expression can be induced in 3-day-old Het pups, 30- $\mu \mathrm{mol}$ heme/kg BW was administered SC, a

Table 1. Hematological profiles of control and CS-only-, Heme-only-, and Heme+CS-treated Het and WT pups

\begin{tabular}{|c|c|c|c|c|c|c|c|c|c|c|c|c|}
\hline & \multicolumn{3}{|l|}{ Control } & \multicolumn{3}{|c|}{ CS-only-treated } & \multicolumn{3}{|c|}{ Heme-only-treated } & \multicolumn{3}{|c|}{ Heme+CS-treated } \\
\hline$W B C s^{a}$ & $4.70 \pm 1.50$ & $5.73 \pm 1.18$ & ns & $3.76 \pm 2.73$ & $2.82 \pm 0.93$ & ns & $6.36 \pm 1.15$ & $8.22 \pm 1.88$ & 0.028 & $2.66 \pm 1.97$ & $2.20 \pm 0.45$ & ns \\
\hline Neutrophils ${ }^{\mathrm{a}}$ & $2.94 \pm 0.47$ & $2.80 \pm 0.78$ & ns & $2.19 \pm 2.63$ & $0.75 \pm 0.51$ & ns & $4.67 \pm 0.75$ & $5.43 \pm 1.15$ & ns & $1.13 \pm 1.58$ & $0.48 \pm 0.14$ & ns \\
\hline Lymphocytes $^{a}$ & $1.50 \pm 1.38$ & $2.57 \pm 0.75$ & 0.029 & $1.35 \pm 0.18$ & $1.85 \pm 0.85$ & ns & $1.45 \pm 0.76$ & $2.42 \pm 1.09$ & ns & $1.31 \pm 0.47$ & $1.48 \pm 0.63$ & ns \\
\hline Platelets $^{\mathrm{b}}$ & $4.10 \pm 1.66$ & $6.92 \pm 2.00$ & 0.040 & $3.15 \pm 2.70$ & $2.04 \pm 0.82$ & ns & $6.11 \pm 1.66$ & $6.75 \pm 0.93$ & ns & $2.09 \pm 1.28$ & $2.07 \pm 0.58$ & ns \\
\hline
\end{tabular}


Table 2. Fold change in liver and spleen gene expression profiles of CS-only- and Heme+CS-treated Het pups compared with those we previously reported for WT pups ${ }^{13}$

\begin{tabular}{|c|c|c|c|c|c|c|c|c|}
\hline \multirow[b]{2}{*}{ Gene } & \multicolumn{2}{|c|}{$\frac{\text { Liver }}{\text { CS-only-treated }}$} & \multicolumn{6}{|l|}{ Spleen } \\
\hline & Fold change & $p$-Value & Fold change & $p$-Value & Fold change & $p$-Value & Fold change & $p$-Value \\
\hline $\mathrm{Ccl} 5$ & -9.64 & 0.003 & -3.24 & 0.099 & -2.01 & 0.002 & -1.79 & 0.242 \\
\hline $\mathrm{Cd} 14$ & -4.78 & 0.002 & -1.56 & 0.271 & 1.47 & 0.239 & -1.04 & 0.614 \\
\hline Cd8a & -4.08 & 0.042 & -1.43 & 0.565 & -4.28 & 0.039 & -1.50 & 0.345 \\
\hline Mpo & 1.42 & 0.325 & 1.13 & 0.668 & 4.45 & 0.048 & 1.28 & 0.426 \\
\hline Ticam 1 & 1.34 & 0.235 & -4.31 & 0.004 & 1.38 & 0.375 & 1.24 & 0.560 \\
\hline Tnf & -1.78 & 0.149 & -5.30 & 0.033 & -1.69 & 0.101 & -1.15 & 0.565 \\
\hline TIr5 & 6.86 & 0.009 & 2.07 & 0.075 & -1.12 & 0.756 & 1.67 & 0.246 \\
\hline Ifng & -52.56 & 0.005 & -18.58 & 0.021 & -6.26 & 0.060 & -3.98 & 0.131 \\
\hline
\end{tabular}

\begin{tabular}{|c|c|c|c|c|c|c|c|c|}
\hline & \multicolumn{2}{|l|}{ Liver } & \multicolumn{2}{|l|}{ Spleen } & \multicolumn{2}{|l|}{ Liver } & \multicolumn{2}{|l|}{ Spleen } \\
\hline Gene & Fold change & $p$-Value & Fold change & $p$-Value & Fold change & $p$-Value & Fold change & $p$-Value \\
\hline $\mathrm{Ccl} 12$ & 2.12 & 0.043 & 5.09 & 0.042 & 2.61 & 0.198 & 10.90 & 0.005 \\
\hline $\mathrm{Cd} 4$ & -2.69 & 0.027 & -1.60 & 0.142 & -6.90 & 0.010 & -1.13 & 0.783 \\
\hline Cxcr3 & -6.82 & 0.007 & -2.35 & 0.029 & -10.31 & 0.002 & -2.97 & 0.020 \\
\hline Cxcl10 & 35.67 & 0.001 & 15.47 & 0.001 & 28.20 & 0.004 & 24.99 & 0.006 \\
\hline Icam1 & 18.17 & 0.005 & 4.74 & 0.018 & 13.59 & 0.003 & 7.16 & 0.013 \\
\hline Il10 & 60.97 & 0.170 & 11.40 & 0.050 & 7.45 & 0.078 & 5.00 & 0.038 \\
\hline II1a & 3.70 & 0.023 & 8.16 & 0.011 & 2.89 & 0.010 & 9.62 & 0.073 \\
\hline Myd88 & 6.57 & 0.027 & 2.69 & 0.014 & 4.19 & 0.003 & 3.09 & 0.055 \\
\hline Nfkbia & 18.47 & 0.015 & 9.31 & 0.007 & 12.08 & $>0.001$ & 8.30 & 0.007 \\
\hline Nlrp3 & 4.54 & 0.035 & 4.33 & 0.013 & 2.47 & 0.093 & 4.50 & 0.030 \\
\hline Tlr2 & 10.08 & 0.002 & 5.97 & $>0.001$ & 9.05 & 0.003 & 6.15 & 0.001 \\
\hline Tlr5 & -1.52 & 0.100 & -4.34 & 0.002 & -4.01 & 0.002 & -2.89 & 0.016 \\
\hline Tnf & 5.75 & 0.004 & 4.39 & 0.002 & 1.60 & 0.027 & 5.10 & 0.006 \\
\hline
\end{tabular}

dose we have previously observed to cause a significant 1.5 -fold induction of HO-1 expression in WT pups. ${ }^{19}$ At $24 \mathrm{~h}$ post-treatment, liver $\mathrm{HO}$ activity in Heme-only-treated pups significantly increased by $58 \%(335 \pm 39 \mathrm{pmol} \mathrm{CO} / \mathrm{h} / \mathrm{mg} \mathrm{FW}, n=15, p<0.01$, Fig. 3) compared with age-matched control Het pups and similar to baseline WT levels, but not to the level of Heme-only-treated WT pups $(496 \pm 85 \mathrm{pmol} \mathrm{CO} / \mathrm{h} / \mathrm{mg} \mathrm{FW}, n=10, p<0.01)$. In addition, at $24 \mathrm{~h}$ post-sepsis induction, liver $\mathrm{HO}$ activity of Heme+CS-treated pups also increased $(319 \pm 38 \mathrm{pmol} \mathrm{CO} / \mathrm{h} / \mathrm{mg} \mathrm{FW}, n=7, p<0.01$, Fig. 3) compared with control Het pups, but not to the level of Heme + CS-treated WT pups ( $459 \pm 47 \mathrm{pmol} \mathrm{CO} / \mathrm{h} / \mathrm{mg} \mathrm{FW}, n=24, p<0.01)$.
Hematology. Although there were no significant differences in WBCs, lymphocytes, and platelets in Heme-only-treated Het pups compared with control Het pups, neutrophils significantly increased $(p<0.005$, Table 1$)$. On the other hand, there were no significant differences in neutrophils, lymphocytes, and platelets in Heme-only-treated Het compared with Heme-only-treated WT pups, but WBCs in Heme-only-treated Het pups were significantly lower than those in Heme-only-treated WT pups.

In Heme+CS-treated Het pups, WBCs, neutrophils, lymphocytes, or platelets did not significantly change compared with those of CS-only-treated Het pups. 
Table 4. Fold change in gene expression profiles of the livers of Heme-only-treated Het pups compared with control Het pups

\begin{tabular}{lll}
\hline \multicolumn{3}{c}{ Heme-only-treated/controls } \\
\hline Gene & Fold change & $p$-Value \\
Ccl12 & $-\mathbf{4 . 9 9}$ & $\mathbf{0 . 0 0 2}$ \\
Cd4 & $-\mathbf{5 . 3 6}$ & $\mathbf{0 . 0 1 0}$ \\
\hline
\end{tabular}

Fold change $>4$ is significant

Table 5. Fold change in gene expression profiles of the liver and spleens of Heme+CS-treated Het pups compared with CS-only-treated Het pups

\begin{tabular}{|c|c|c|c|c|}
\hline \multirow[b]{2}{*}{ Gene } & \multicolumn{2}{|l|}{ Liver } & \multicolumn{2}{|l|}{ Spleen } \\
\hline & Fold change & $p$-Value & Fold change & $p$-Value \\
\hline II23a & -5.14 & 0.041 & 1.31 & 0.920 \\
\hline Irf7 & -4.08 & 0.011 & -1.17 & 0.568 \\
\hline Ticam1 & -5.03 & 0.011 & -1.37 & 0.385 \\
\hline
\end{tabular}

Gene expression profiles. In Heme-only-treated Het pups, Ccl12 (-5.0-fold) and Cd4 (-5.4-fold) were significantly reduced compared with control Het pups ( $p<0.05$, Table 4).

In the livers of Heme+CS-treated Het pups, most of the upregulated genes were downregulated. Particularly, one cytokine (II23a) and two other genes related to the immune response (Irf7, Ticam 1) were significantly downregulated (>4-fold) compared with CS-only-treated Het pups (Table 5).

In Heme+CS-treated Het pups, gene expression of Ticam 1 (-4.3-fold), Tnf (-5.3-fold), and Ifng (-18.6-fold) in the livers were significantly reduced compared with that of WT pups, and no genes significantly changed in the spleens (Table 2 ).

\section{DISCUSSION}

The treatment of sepsis in preterm infants still remains a challenge, as the mortality rate from sepsis is much higher than those of term infants or adults. ${ }^{24}$ This suggests that immature protective mechanisms are present in the vulnerable preterm population. It is known that HO-1 is a stress-response protein that is induced by a variety of factors such as heme, heavy metals, endotoxin, inflammatory cytokines, and prostaglandins, ${ }^{25}$ and thought to play a central role in regulating the inflammatory response. ${ }^{26}$ In fact, during the perinatal period, $\mathrm{HO}-1$ expression in the liver and lung are maximally expressed, implying a physiological and pathological importance of this heat-shock protein. $^{13}$ Thus, we speculated that a deficiency in HO-1 expression during sepsis can be detrimental and even lethal, especially in immunodeficient preterm infants. To date, only a few number of studies have investigated the role of HO-1 deficiency in sepsis, with none specifically focusing on newborn pups. To this end, we applied our non-surgical CS model ${ }^{13}$ to HO-1-deficient (Het) newborn pups to investigate the effect of a partial deficiency in HO-1 on the severity of sepsis.

Using an adult sepsis model, Chung et al. reported that mice with a complete $\mathrm{HO}-1$ deficiency $\left(\mathrm{HO}-1^{-/-}\right)$had a higher mortality rate compared with WT mice. They also showed in a transgenic mouse that the overexpression of $\mathrm{HO}-1$ in the vasculature had improved survival after sepsis induction. ${ }^{7}$ Larsen et al. reported that a higher rate of sepsis-induced mortality occurred in $\mathrm{HO}-1^{-1-}$ mice and speculated that this was due to increased levels of circulating free heme and reduced levels of hemopexin, which resulted in an increase in oxidative stress and tissue damage. ${ }^{8,27}$ In contrast, Czaikoski et al. ${ }^{10}$ demonstrated an improved survival rate in septic adult mice given zinc deuteroporphyrin 2,4-bis glycol (ZnBG) to inhibit HO. None of these studies, however, reported significant differences in mortality due to sepsis between Het and WT adult mice. ${ }^{7}$ Although an improved survival rate was found by Czaikoski et al. ${ }^{10}$ after inhibition of HO-1 and in contrast to our findings, we speculate that their observation may be due to an upregulation of $\mathrm{HO}-1$ by $\mathrm{ZnBG}$. We have shown that despite being a short-acting $\mathrm{HO}$ inhibitor, $\mathrm{ZnBG}$ is not specific for the inducible $\mathrm{HO}-1^{28}$ and can also induce HO-1 expression at doses found to inhibit $\mathrm{HO}$ activity. ${ }^{18}$ Because of the uncontrollable ying/yang effects of certain metalloporphyrins, using an HO-1-deficient mouse model can eliminate these potential confounding factors.

In our study, we found that liver $\mathrm{HO}$ activity was lower in Het compared with WT pups as expected. Interestingly, lymphocyte and platelet numbers were significantly lower at basal levels, suggesting an impaired hematopoiesis in HO-1 deficiency. When gene expression profiles were performed, no significant differences were found between Het and WT pups at baseline.

Following sepsis induction, the mortality rate of 4-day-old Het pups was significantly higher (85.0\%) at the LD40 we previously established for WT pups. ${ }^{13}$ In addition, Het mice showed an increase of HO-1 expression post-heme or sepsis induction, but not as high as that of the WT pups. On the other hand, hematological parameters and organ bacterial counts were comparable to those of the WT pups. In addition, most upregulated genes in the livers and spleens of post-septic Het pups were the same genes upregulated in post-septic WT pups as previously reported. We speculate that a normal expression of $\mathrm{HO}-$ 1 following sepsis induction might be critical for survival during the neonatal period.

In the livers of CS-only-treated Het pups, the genes associated with the immune response against bacteria $(\mathrm{C} C \mathrm{Cl}, \mathrm{Cd} 14, \mathrm{Cd} 8 \mathrm{a}$, and Ifng) were downregulated, and only T/r5, a pathogen-recognition receptor, was upregulated. In the spleens of CS-only-treated Het pups, $C d 8 a$ was significantly downregulated similar to the livers. Genes associated with inflammation (I/6) and oxidative stress (Mpo) were upregulated compared with those in CS-only-treated WT pups. In particular, Ifng mRNA levels were downregulated $>50$ fold in CS-only-treated Het pups compared to CS-only-treated WT pups. Several in vitro studies have found strong associations between Ifng and HO-1. Takahashi et al. ${ }^{29}$ reported that in human glioblastoma cells, HO-1 mRNA expression was suppressed by Ifng exposure. In the other study, Kovacsics et al. ${ }^{30}$ observed that HO-1 protein expression was reduced in astrocytes that were treated with Ifng. In addition, Singh et al. ${ }^{31}$ demonstrated that Ifnginduced autophagy is dependent upon HO-1-mediated signaling. Thus, we speculate that the significant downregulation of Ifng that we observed in Het pups suggests that there is a dysregulation of Ifng following sepsis. These findings also suggest that a reduced immune response and an increase in systemic inflammation during sepsis contributes to the increased mortality found in Het pups. In humans, there exist two functional polymorphisms in the HO-1 promoter region, which have been shown to affect HO-1 expression, $A(-413) T$, and (GT)n repeats. They have been studied extensively and shown to be associated with a variety of diseases. $^{32}$ Individuals possessing the -413 AA genotype have increased HO-1 expression. ${ }^{33}$ The presence of short (GT)n repeats has been correlated with an increased HO-1 expression and reported as being preventive in human pediatric sepsis. ${ }^{12}$ In contrast, low HO-1 expression caused by these polymorphisms (such as long (GT)n repeats) is associated with a number of diseases. $^{34}$ Our HO-1 Het mice, which have low basal HO-1 expression and reduced $\mathrm{HO}-1$ inducibility, reflect humans with functional HO-1 polymorphisms (such as long (GT)n repeats or $-413 \mathrm{TT}$ genotype), that result in a reduction of $\mathrm{HO}-1$ induction. ${ }^{32}$ 
To investigate whether $\mathrm{HO}-1$ is protective in sepsis, we evaluated the liver gene expression and hematological profiles $24 \mathrm{~h}$ post-heme administration in Het pups. We found that heme treatment overall attenuated cytokines and immune-modulating genes in the liver and significantly induced circulating neutrophils. Interestingly, $\mathrm{Ccl} 12$ and $\mathrm{Ccr} 4$ were significantly downregulated in the liver post heme treatment. These genes are both involved in cytokine-induced immune and inflammatory responses, which might prevent the progression of systemic inflammation caused by sepsis, and thus, be protective by mediating immune and chemotactic function.

When we treated Het pups with heme $24 \mathrm{~h}$ prior to sepsis induction, the mortality rate significantly decreased to only $23.5 \%$ (from $85.0 \%$ for CS-only-treated Het pups). This finding was similar to what we had observed in our recent paper using only WT pups, which decreased to $6.3 \%$ for Heme+CS-treated WT pups (from $40.9 \%$ for CS-only-treated WT pups). ${ }^{13}$ In addition, we observed that the upregulation of immune-related genes, especially II23a, Irf7, and Ticam 1, were attenuated in surviving Heme+CS-treated Het pups and was similar to what we previously observed for WT mice. Moreover, the genes associated with innate immunity (Ticam1, Tnf, and Ifng) were downregulated in comparison with Heme+CS-treated WT pups, which suggests a reduced immune response against invading pathogens.

Interestingly, liver HO activity of control WT and Heme-onlytreated Het pups at 4 days of age was similar; however, the survival rate of Heme+CS-treated Het pups (76.5\%) was significantly higher than that of CS-only-treated WT pups (40.9\%). When we compared the gene expression profiles of the livers from control WT pups, we found that only $C \mathrm{cr} 5(-4.3, p<0.01)$ was significantly reduced in Heme-only-treated Het pups. Ccr5 is the main co-receptor of the human immunodeficiency virus and involved in virus entry and cell-to-cell spread. ${ }^{35}$ Interestingly, Ccr5 deficiency is reported to play a protective role in rheumatoid arthritis via inhibiting T-cell migration, a key pathway in the inflammatory process. ${ }^{36,37} \mathrm{Ccr} 5$-mediated cytokine signaling is also involved in the process of acute rejection in transplanted patients via cell recruitment into clinical allografts. ${ }^{38}$ Thus, we hypothesized that Heme+CS-treated Het pups might have a reduction in inflammatory cell migration in comparison with control WT pups, resulting in attenuated post-septic inflammatory reaction, and hence increased survival. Previous reports have also shown that inflammatory responses can be inhibited in $\mathrm{HO}-1$ overexpressing macrophages, ${ }^{39}$ and $\mathrm{HO}-1$ and its metabolites can reduce leukocyte rolling, adhesion, and neutrophil infiltration and may induce a suppressive phenotype such as myeloid-derived suppressor cells. ${ }^{40}$ Further studies investigating the specific protein expression patterns of these immune-related and inflammatory genes are still warranted to fully elucidate the exact mechanism(s) by which HO-1 may upregulate their expression, and it is beyond the scope of this manuscript.

In conclusion, we found that a partial deficiency in HO-1 increases the severity and mortality in Het pups exposed to sepsis. Furthermore, induction of $\mathrm{HO}-1$ in Het pups significantly reduced mortality. This reduction in mortality was associated with an attenuation of the expression of immune-related genes and an increase in peripheral neutrophils. Thus, we conclude that HO-1 may play an important role in the protection against preterm sepsis, and the induction of HO-1 might be a feasible approach for treating sepsis in susceptible newborns.

\section{ACKNOWLEDGEMENTS}

We thank Dr. Hendrik J. Vreman for an invaluable advice on our experimental design. This work was supported by the Christopher Hess Research Fund, the H.M. Lui Research Fund (Hong Kong), the Mary L. Johnson Research Fund, and the Child Health Research Institute at Stanford, and the Kobe Sinryokukai Association (Japan),
JSPS KAKENHI Grant Number 16H06971 (Japan), and the Mother and Child Health Foundation (Japan).

\section{ADDITIONAL INFORMATION}

Competing interests: The authors declare no competing interests.

Publisher's note: Springer Nature remains neutral with regard to jurisdictional claims in published maps and institutional affiliations.

\section{REFERENCES}

1. Wynn, J. L. \& Wong, H. R. Pathophysiology and treatment of septic shock in neonates. Clin. Perinatol. 37, 439-479 (2010).

2. Wynn, J. L. et al. Increased mortality and altered immunity in neonatal sepsis produced by generalized peritonitis. Shock 28, 675-683 (2007).

3. Stoll, B. J. et al. Early onset neonatal sepsis: the burden of group B Streptococcal and E. coli disease continues. Pediatrics 127, 817-826 (2011).

4. Maines, M. D. Heme oxygenase: function, multiplicity, regulatory mechanisms, and clinical applications. FASEB J. 2, 2557-2568 (1988).

5. Tenhunen, R., Marver, H. S. \& Schmid, R. The enzymatic conversion of heme to bilirubin by microsomal heme oxygenase. Proc. Natl Acad. Sci. USA 61, 748-755 (1968).

6. Stocker, R., Yamamoto, Y., Mcdonagh, A. F., Glazer, A. N. \& Ames, B. N. Bilirubin is an antioxidant of possible physiological importance. Science 235, 1043-1046 (1987).

7. Chung, S. W., Liu, X., Macias, A. A., Baron, R. M. \& Perrella, M. A. Heme oxygenase1-derived carbon monoxide enhances the host defense response to microbial sepsis in mice. J. Clin. Invest. 118, 239-247 (2008).

8. Tsoyi, K. et al. Heme-oxygenase- 1 induction and carbon monoxide-releasing molecule inhibit lipopolysaccharide (LPS)-induced high-mobility group box 1 release in vitro and improve survival of mice in LPS- and cecal ligation and puncture-induced sepsis model in vivo. Mol. Pharmacol. 76, 173-182 (2009).

9. Carchman, E. H., Rao, J., Loughran, P. A., Rosengart, M. R. \& Zuckerbraun, B. S. Heme oxygenase-1-mediated autophagy protects against hepatocyte cell death and hepatic injury from infection/sepsis in mice. Hepatology 53, 2053-2062 (2011).

10. Czaikoski, P. G. et al. Heme oxygenase inhibition enhances neutrophil migration into the bronchoalveolar spaces and improves the outcome of murine pneumonia-induced sepsis. Shock 39, 389-396 (2013).

11. Takaki, S. et al. Beneficial effects of the heme oxygenase-1/carbon monoxide system in patients with severe sepsis/septic shock. Intensive Care. Med. 36, 42-48 (2010).

12. Vazquez-Armenta, G. et al. Short (GT)n microsatellite repeats in the heme oxygenase-1 gene promoter are associated with antioxidant and antiinflammatory status in Mexican pediatric patients with sepsis. Tohoku. J. Exp. Med. 231, 201-209 (2013).

13. Fujioka, K. et al. Induction of heme oxygenase- 1 attenuates the severity of sepsis in a non-surgical preterm mouse model. Shock 47, 242-250 (2017).

14. Starr, M. E. et al. A new cecal slurry preparation protocol with improved long-term reproducibility for animal models of sepsis. PLOS ONE 9, e115705 (2014).

15. Steele, A. M., Starr, M. E. \& Saito, H. Late therapeutic intervention with antibiotics and fluid resuscitation allows for a prolonged disease course with high survival in a severe murine model of sepsis. Shock 47, 726-734 (2017).

16. Adkins, B., Leclerc, C. \& Marshall-Clarke, S. Neonatal adaptive immunity comes of age. Nat. Rev. Immunol. 4, 553-564 (2004).

17. Zhao, H., Wong, R. J., Kalish, F. S., Nayak, N. R. \& Stevenson, D. K. Effect of heme oxygenase-1 deficiency on placental development. Placenta 30, 861-868 (2009).

18. He, C. X. et al. Effects of zinc deuteroporphyrin bis glycol on newborn mice after heme-loading. Pediatr. Res. 70, 467-472 (2011).

19. Fujioka, K., Kalish, F., Wong, R. J. \& Stevenson, D. K. Inhibition of heme oxygenase activity using a microparticle formulation of zinc protoporphyrin in an acute hemolytic newborn mouse model. Pediatr. Res. 79, 251-257 (2016).

20. Kronforst, K. D. et al. A neonatal model of intravenous Staphylococcus epidermidis infection in mice $24 \mathrm{~h}$ old enables characterization of early innate immune responses. PLOS ONE 7, e43897 (2012).

21. Ge, Y. et al. Relationship of tissue and cellular interleukin-1 and lipopolysaccharide after endotoxemia and bacteremia. J. Infect. Dis. 176, 1313-1321 (1997).

22. Borish, L. C. \& Steinke, J. W. 2. Cytokines and chemokines. J. Allergy Clin. Immunol. 111, S460-S475 (2003).

23. Rot, A. \& von Andrian, U. H. Chemokines in innate and adaptive host defense: basic chemokinese grammar for immune cells. Annu. Rev. Immunol. 22, 891-928 (2004). 
24. Stoll, B. J. \& Fanaroff, A. Early-onset coagulase-negative staphylococcal sepsis in preterm neonate. National Institute of Child Health and Human Development (NICHD) Neonatal Research Network. Lancet 345, 1236-1237 (1995).

25. Wang, R. et al. Stress-responsive heme oxygenase-1 isoenzyme participates in Toll-like receptor 4-induced inflammation during brain ischemia. Neuroreport 27, 445-454 (2016)

26. Liong, S. \& Lappas, M. The stress-responsive heme oxygenase (HO)-1 isoenzyme is increased in labouring myometrium where it regulates contraction-associated proteins. Am. J. Reprod. Immunol. 74, 62-76 (2015).

27. Larsen, R. et al. A central role for free heme in the pathogenesis of severe sepsis. Sci. Transl. Med 2, 51 ra71 (2010).

28. Wong, R. J. et al. In vitro inhibition of heme oxygenase isoenzymes by metalloporphyrins. J. Perinatol. 31(Suppl 1), S35-S41 (2011).

29. Takahashi, K., Nakayama, M., Takeda, K., Fujia, H. \& Shibahara, S. Suppression of heme oxygenase-1 mRNA expression by interferon-gamma in human glioblastoma cells. J. Neurochem. 72, 2356-2361 (1999).

30. Kovacsics, C. E., Gill, A. J., Ambegaokar, S. S., Gelman, B. B. \& Kolson, D. L. Degradation of heme oxygenase- 1 by the immunoproteasome in astrocytes: a potential interferon-gamma-dependent mechanism contributing to HIV neuropathogenesis. Glia 65, 1264-1277 (2017).

31. Singh, N. et al. Antimycobacterial effect of IFNG (interferon gamma)-induced autophagy is dependent on the HMOX1 (heme oxygenase 1)-mediated increase in the intracellular calcium levels and modulation of PPP3/calcineurin-TFEB (transcription factor EB) axis. Autophagy (2018). In press. https://doi.org/10.1080/ 15548627.2018.1436936.
32. Fujioka, K. et al. Heme oxygenase-1 promoter polymorphisms and risk of spina bifida. Birth Defects Res. A Clin. Mol. Teratol. 103, 741-746 (2015).

33. Ono, K. et al. A promoter variant of the heme oxygenase- 1 gene may reduce the incidence of ischemic heart disease in Japanese. Atherosclerosis 173, 315-319 (2004)

34. Exner, M., Minar, E., Wagner, O. \& Schillinger, M. The role of heme oxygenase-1 promoter polymorphisms in human disease. Free Radic. Biol. Med. 37, 1097-1104 (2004).

35. Lopalco, L. CCR5: from natural resistance to a new anti-HIV strategy. Viruses 2, 574-600 (2010).

36. Prahalad, S. Negative association between the chemokine receptor CCR5-Delta32 polymorphism and rheumatoid arthritis: a meta-analysis. Genes Immun. 7, 264-268 (2006).

37. Yang, Y. F. et al. A non-peptide CCR5 antagonist inhibits collagen-induced arthritis by modulating $\mathrm{T}$ cell migration without affecting anti-collagen $\mathrm{T}$ cell responses. Eur. J. Immunol. 32, 2124-2132 (2002).

38. Schroder, C. et al. CCR5 blockade modulates inflammation and alloimmunity in primates. J. Immunol. 179, 2289-2299 (2007).

39. Minamino, T. et al. Targeted expression of heme oxygenase- 1 prevents the pulmonary inflammatory and vascular responses to hypoxia. Proc. Natl Acad. Sci. USA 98, 8798-8803 (2001).

40. Schumacher, A. \& Zenclussen, A. C. Effects of heme oxygenase-1 on innate and adaptive immune responses promoting pregnancy success and allograft tolerance. Front. Pharmacol. 5, 288 (2014). 